\title{
A NOTE ON THE FUSION OF TWO VERTICES IN A FUZZY GRAPH
}

\author{
By \\ P. V. RAMAKRISHNAN and T. LAKSHMI
}

\begin{abstract}
The concept of fuzzy sets was introduced by L. A. Zadeh in 1965. The concept of fuzzy graph was introduced by A. Rosenfeld [5] in 1975. Many of the crisp graph concepts have been extended to fuzzy graph theory. Here we define the fusion of two vertices in a fuzzy graph and investigate some properties and also give fusion algorithm for effective connectedness and for adjacency matrix.
\end{abstract}

\section{Preliminaries}

A fuzzy graph $G=(\sigma, \mu)$ is a pair of functions $\sigma: S \rightarrow[0,1]$ and $\mu: S X S \rightarrow[0,1]$ such that for all $x, y$ in $S$ we have $\mu(x, y) \leq \sigma(x) \wedge \sigma(y)$ where $S$ is the underlying (vertex) set. (In this paper we always consider $S$ as a finite set.) For the fuzzy graph $G, G^{*}=\left(\sigma^{*}, \mu^{*}\right)$ is called the corresponding (crisp) support graph where $\sigma^{*}=$ Support of $\sigma$ and $\mu^{*}=$ Support of $\mu$. The fuzzy graph $H=(\tau, v)$ is called a (partial) fuzzy subgraph of $G$ if $\tau(x) \leq \sigma(x)$ and $v(x, y) \leq \mu(x, y)$ for all $x, y$ in $S$. The fuzzy graph $H=(\tau, v)$ is called a spanning fuzzy subgraph of $G$ if $\tau(x)=\sigma(x)$ for all $x$ in $S$. A fuzzy graph $G=(\sigma, \mu)$ is called a complete fuzzy graph if $\mu(x, y)=\sigma(x) \wedge \sigma(y)$ for all $x$ and $y$; it is called quasi complete if $G^{*}$ is complete (clearly complete implies quasi complete). A complete fuzzy graph with $n$ vertices is denoted by $\sigma_{n}$.

We shall call a fuzzy graph $G=(\sigma, \mu)$, bipartite* if the graph $G^{*}=\left(\sigma^{*}, \mu^{*}\right)$ is bipartite where $\sigma^{*}=\operatorname{Supp} \sigma$ and $\mu^{*}=\operatorname{Supp} \mu$. We call a fuzzy graph $G=(\sigma, \mu)$, fuzzy bipartite* if it has a spanning fuzzy subgraph $F=(\sigma, v)$ which is bipartite* and for all arcs $(x, y)$ not in $F$ (i.e. $v(x, y)=0$ ), we have, $\mu(x, y)<$ $v^{\infty}(x, y)$.

A fuzzy graph $G=(\sigma, \mu)$ is called complete bipartite* if $G^{*}=\left(\sigma^{*}, \mu^{*}\right)$ is a complete bipartite graph. $G$ is called complete fuzzy bipartite* fuzzy graph if $G$

Key words. Fuzzy graph, complete fuzzy graph, fusion of vertices in a graph, adjacency matrix. Received June 28, 2007. 
has a spanning fuzzy subgraph $F=\left(\sigma, \mu^{\prime}\right)$ which is complete bipartite* and for all arcs $(x, y)$ not in $F, \mu(x, y)<\mu^{\infty}(x, y)$.

An edge $(x, y)$ of a fuzzy graph $G=(\sigma, \mu)$ is called an effective edge if $\mu(x, y)=\sigma(x) \wedge \sigma(y)$. A path in $G$ is called an effective path if all the edges in that path are effective edges. A pair of vertices of $G$ are called effectively connected if there is an effective path between them. A fuzzy graph is called effectively connected if every pair of vertices are effectively connected.

The terms undefined here may be seen in [1], [2] \& [5].

\section{Fusion of Vertices in A Fuzzy Graph}

Definition 2.1. A fuzzy graph $G=(\sigma, \mu)$ is called strong complete fuzzy

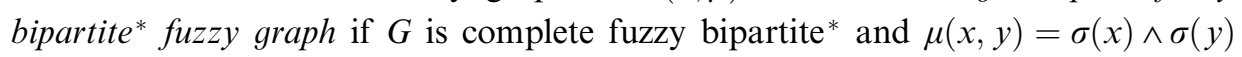
for all $(x, y)$ in $F$ and it is denoted by $\sigma_{m, n}$.

EXAMPLE 2.2.
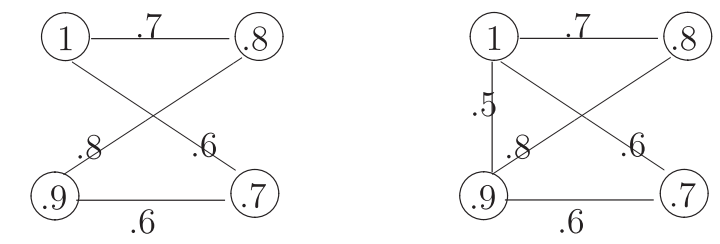

complete bipartite* $\quad$ complete fuzzy bipartite*

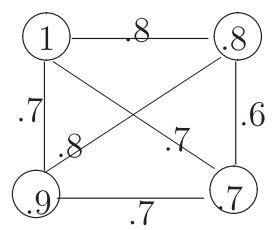

strong complete fuzzy bipartite*

Definition 2.3. Let $G=(\sigma, \mu)$ be a fuzzy graph and let $u, v \in \sigma^{*}$. By the fusion of two vertices $u$ and $v$ we mean the following:

(i) Fuse the vertices $u$ and $v$ as $u v$ in the corresponding crisp graph $G^{*}=\left(\sigma^{*}, \mu^{*}\right)$ and then consider its underlying simple graph.

(ii) The resulting fuzzy graph is $G_{u v}=\left(\sigma_{u v}, \mu_{u v}\right)$ where 
A note on the fusion of two vertices in a fuzzy graph

$$
\sigma_{u v}(x)= \begin{cases}\max [\sigma(u), \sigma(v)], & \text { if } x=u v \\ \sigma(x), & \text { if } x \neq u v\end{cases}
$$

and

$$
\mu_{u v}(x, y)= \begin{cases}\max [\mu(x, u), \mu(x, v)], & \text { if } y=u v \\ \max [\mu(u, y), \mu(v, y)], & \text { if } x=u v \\ \mu(x, y), & \text { if } x \neq u v \text { and } y \neq u v\end{cases}
$$

EXAMPLE 2.4 .
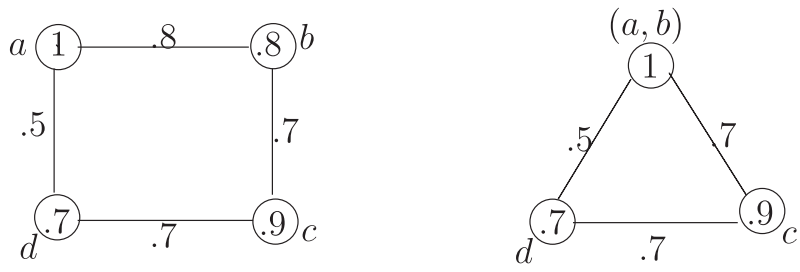

$$
G=(\sigma, \mu)
$$

$$
G_{a b}
$$

Proposition 2.5. The fusion of two vertices of $K_{n}$ results in $K_{n-1}$.

The analogous result in fuzzy graphs is as follows:

Proposition 2.6. The fusion of two vertices of a complete fuzzy graph on $n$ vertices results in a complete fuzzy graph.

(i.e.) The fusion of two vertices of $\sigma_{n}$ results in $\sigma_{n-1}$.

Proof. Let $G=(\sigma, \mu)$ be a complete fuzzy graph with $n$ vertices. (i.e.) $G=\sigma_{n}$. Suppose $x$ and $y$ are fused together and get $G_{x y}=\left(\sigma_{x y}, \mu_{x y}\right)$. By definition, $\sigma_{x y}(x, y)=\operatorname{Max}[\sigma(x), \sigma(y)]$. Without loss of generality assume that $\sigma(x)<\sigma(y)$. Therefore, $\sigma_{x y}(x y)=\sigma(y)$. Suppose $z$ is any vertex other than $x y$ in $G_{x y}$. Hence $\sigma_{x y}(z)=\sigma(z)$. Then

$$
\begin{aligned}
\mu_{x y}(x y, z) & =\operatorname{Max}[\mu(x, z), \mu(y, z)] \\
& =\operatorname{Max}[\sigma(x) \wedge \sigma(z), \sigma(y) \wedge \sigma(z)] \\
& =(\sigma(x) \wedge \sigma(z)) \vee(\sigma(y) \wedge \sigma(z)) \\
& =(\sigma(x) \vee \sigma(y)) \wedge \sigma(z) \\
& =\sigma_{x y}(x y) \wedge \sigma_{x y}(z)
\end{aligned}
$$


By definition,

$$
\begin{aligned}
\mu_{x y}(u, v) & =\mu(u, v) \text { for all } u, v \text { where } u \neq(x, y) \text { and } v \neq(x, y) \\
& =\sigma(u) \wedge \sigma(v) \\
& =\sigma_{x y}(u) \wedge \sigma_{x y}(v)
\end{aligned}
$$

Hence $G_{x y}=\sigma_{n-1} . \quad \diamond$

Proposition 2.7. The fusion of any two vertices of $K_{1, n}$ results in $K_{1, n-1}$.

The above result need not be true in fuzzy graphs. The fusion of two vertices of $\sigma_{1, n}$ with both vertices in $V_{1}$ or in $V_{2}$ results in $\sigma_{1, n-1}$; fusion with one vertex in $V_{1}$ and another in $V_{2}$ need not result in $\sigma_{1, n-1}$. (i.e.)

Proposition 2.8. The fusion of two vertices of $\sigma_{1, n}$ with both the vertices in same set $\left(V_{1}\right.$ or $\left.V_{2}\right)$ results in $\sigma_{1, n-1}$.

EXAMPLE 2.9.

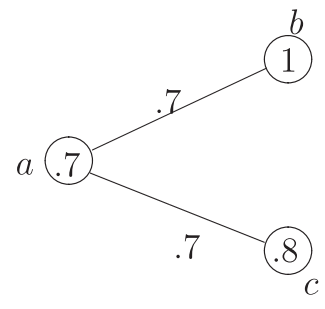

G

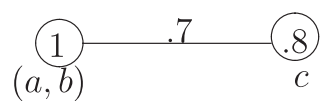

$G_{a b}$

Clearly $G=\sigma_{1,2}$ but $G_{a b} \neq \sigma_{1,1}$.

Proposition 2.10. The fusion of two vertices of $\sigma_{1, n}$ with one vertex in $V_{1}$ and another in $V_{2}$ results in $\sigma_{1, n-1}$ iff for every pair of vertices $u_{2}$ and $v_{2}$ of $V_{2}$,

$$
\sigma\left(v_{1}\right) \geq \operatorname{Min}\left[\sigma\left(u_{2}\right), \sigma\left(v_{2}\right)\right] \text { where } V_{1}=\left\{v_{1}\right\}
$$


ProOF.

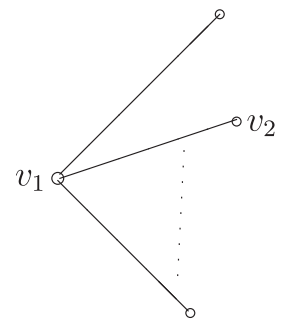

$\sigma_{1, n}$

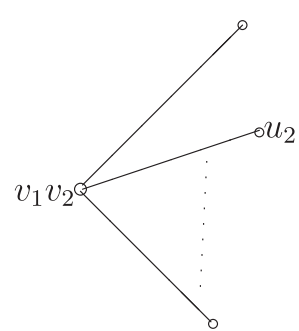

$\sigma_{1, n-1}$

Suppose the inequality (1) is true. Let $v_{1}$ be fused with $v_{2}$. Then $\sigma_{v_{1} v_{2}}\left(v_{1} v_{2}\right)=\operatorname{Max}\left[\sigma\left(v_{1}\right), \sigma\left(v_{2}\right)\right]$. Let $u_{2}$ be any vertex other than $v_{2}$ in $V_{2}$. Then

$$
\begin{aligned}
\mu_{v_{1} v_{2}}\left(v_{1} v_{2}, u_{2}\right) & =\mu\left(v_{1}, u_{2}\right) \\
& =\sigma\left(v_{1}\right) \wedge \sigma\left(u_{2}\right)
\end{aligned}
$$

Case-(i)

Suppose $\sigma\left(u_{2}\right)<\sigma\left(v_{1}\right)<\sigma\left(v_{2}\right)$. Then (2) implies

$$
\begin{aligned}
\mu_{v_{1} v_{2}}\left(v_{1} v_{2}, u_{2}\right) & =\sigma\left(u_{2}\right) \\
& =\sigma_{v_{1} v_{2}}\left(u_{2}\right) \\
& =\sigma_{v_{1} v_{2}}\left(v_{1} v_{2}\right) \wedge \sigma_{v_{1} v_{2}}\left(u_{2}\right)
\end{aligned}
$$

Case-(ii)

Suppose $\sigma\left(v_{2}\right)<\sigma\left(v_{1}\right)<\sigma\left(u_{2}\right)$. Then (2) implies

$$
\begin{aligned}
\mu_{v_{1} v_{2}}\left(v_{1} v_{2}, u_{2}\right) & =\sigma\left(v_{1}\right) \\
& =\sigma_{v_{1} v_{2}}\left(v_{1} v_{2}\right) \wedge \sigma_{v_{1} v_{2}}\left(u_{2}\right)
\end{aligned}
$$

Case-(iii)

Suppose $\sigma\left(u_{2}\right)<\sigma\left(v_{2}\right)<\sigma\left(v_{1}\right)$. Then (2) implies

$$
\begin{aligned}
\mu_{v_{1} v_{2}}\left(v_{1} v_{2}, u_{2}\right) & =\sigma\left(u_{2}\right) \\
& =\sigma_{v_{1} v_{2}}\left(u_{2}\right) \\
& =\sigma_{v_{1} v_{2}}\left(v_{1} v_{2}\right) \wedge \sigma_{v_{1} v_{2}}\left(u_{2}\right)
\end{aligned}
$$


Case-(iv)

Suppose $\sigma\left(v_{2}\right)<\sigma\left(u_{2}\right)<\sigma\left(v_{1}\right)$. Then (2) implies

$$
\begin{aligned}
\mu_{v_{1} v_{2}}\left(v_{1} v_{2}, u_{2}\right) & =\sigma\left(u_{2}\right) \\
& =\sigma_{v_{1} v_{2}}\left(u_{2}\right) \\
& =\sigma_{v_{1} v_{2}}\left(v_{1} v_{2}\right) \wedge \sigma_{v_{1} v_{2}}\left(u_{2}\right)
\end{aligned}
$$

Hence in all cases, we have $G_{v_{1} v_{2}}$ is strong complete fuzzy bipartite*. Therefore $G_{v_{1} v_{2}}=\sigma_{1, n-1}$.

Conversely, suppose the fusion of two vertices of $\sigma_{1, n}$ with one vertex in $V_{1}$ and another in $V_{2}$ results in $\sigma_{1, n-1}$.

To prove that $(1)$ is true. Suppose not. Then,

$$
\sigma\left(v_{1}\right)<\operatorname{Min}\left[\sigma\left(u_{2}\right), \sigma\left(v_{2}\right)\right] \text { for some pair of vertices } u_{2}, v_{2} \text { of } V_{2} \text {. }
$$

Without loss of generality, assume that

$$
\operatorname{Min}\left[\sigma\left(u_{2}\right), \sigma\left(v_{2}\right)\right]=\sigma\left(u_{2}\right)
$$

From (3), $\sigma\left(v_{1}\right)<\sigma\left(u_{2}\right) \leq \sigma\left(v_{2}\right)$. If we fuse $u_{2}$ with $v_{1}$ then

$$
\begin{aligned}
\sigma_{v_{1} u_{2}}\left(v_{1} u_{2}\right) & =\sigma\left(u_{2}\right) \\
\mu_{v_{1} u_{2}}\left(v_{1} u_{2}, v_{2}\right) & =\mu\left(v_{1}, v_{2}\right) \\
& =\sigma\left(v_{1}\right) \wedge \sigma\left(v_{2}\right) \\
& =\sigma\left(v_{1}\right) \\
& <\sigma\left(u_{2}\right) \\
& =\sigma_{v_{1} u_{2}}\left(v_{1} u_{2}\right) \wedge \sigma_{v_{1} u_{2}}\left(v_{2}\right)
\end{aligned}
$$

Which is a contradiction. Hence (1) is true.

Proposition 2.11. If $m \neq 1 \neq n$, then the fusion of two vertices of $K_{m, n}$ with both vertices in $V_{1}$ or in $V_{2}$ results in $K_{m-1, n}$ or $K_{m, n-1}$.

The analogous result in fuzzy graphs is as follows:

Proposition 2.12. The fusion of two vertices of $\sigma_{m, n}(m \neq 1 \neq n)$ with both the vertices in $V_{1}$ or in $V_{2}$ results in $\sigma_{m-1, n}$ or $\sigma_{m, n-1}$.

The proof is as similar as that of Proposition 2.6. 
In crisp graph theory, there is an algorithm to compute the adjacency matrix of the resulting graph after fusing two adjacent vertices. In a similar way we give algorithm to compute the adjacency matrix of a fuzzy graph after fusion of two adjacent vertices.

\section{Algorithm to compute the adjacency matrix of a fuzzy graph after fusion of two adjacent vertices}

Let $G=(\sigma, \mu)$ be a fuzzy graph whose adjacency matrix [1] is denoted by $A(G)$. Suppose $u$ and $v$ are adjacent. (i.e.) $\mu(u, v)>0$. Suppose we fuse $u$ and $v$. Then the adjacency matrix of $G_{u v}$ is denoted by $A\left(G_{u v}\right)$ and it can be obtained by the following steps:

\section{Step-1}

Change $u$ 's row to the maximum of $u$ 's row and $v$ 's row and change $u$ 's column to the maximum of $u$ 's column and $v$ 's column.

\section{Step-2}

Delete the row and column corresponding to $v$.

\section{Step-3}

Name $u$ 's row and $u$ 's column as $u v$ and put the entry as 0 corresponding to $(u v, u v)$.

Then the resulting matrix is the adjacency matrix of the new fuzzy graph $G_{u v}$.

Illustration

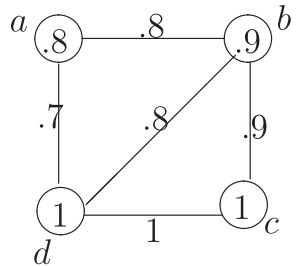

$G$

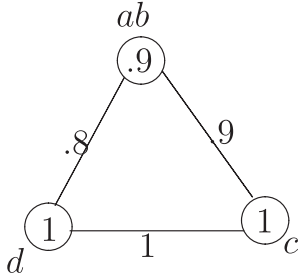

$$
G_{a b}
$$

$$
A(G)=\begin{gathered}
a \\
b \\
c \\
d
\end{gathered}\left(\begin{array}{ccccc}
0 & .8 & 0 & .7 & .8 \\
.8 & 0 & .9 & .8 & .9 \\
0 & .9 & 0 & 1 & 1 \\
.7 & .8 & 1 & 0 & 1
\end{array}\right)
$$


Step-1

$a$
$b$
$c$
$c$
$d$$\left(\begin{array}{lllll}a & b & c & d & \sigma \\ .8 & .8 & .9 & .8 & .9 \\ .8 & 0 & .9 & .8 & .9 \\ .9 & .9 & 0 & 1 & 1 \\ .8 & .8 & 1 & 0 & 1\end{array}\right)$

Step-2

$$
\begin{gathered}
a \\
c \\
d \\
d
\end{gathered}\left(\begin{array}{llll}
.8 & c & d & \sigma \\
.9 & 0 & .8 & .9 \\
.8 & 1 & 0 & 1
\end{array}\right)
$$

Step-3

$$
A\left(G_{a b}\right)=\begin{gathered}
a b \\
c \\
d
\end{gathered}\left(\begin{array}{cccc}
0 & c & d & \sigma \\
.9 & 0 & 1 & 1 \\
.8 & 1 & 0 & 1
\end{array}\right)
$$

The fusion algorithm for effective connectedness

Let $G=(\sigma, \mu)$ be a fuzzy graph whose adjacency matrix is denoted by $A(G)$.

\section{Step-1}

Fuse vertex $v_{1}$ to the first of the vertices $v_{2}, v_{3}, \ldots v_{n}$ with which it is effectively adjacent. In which the new fuzzy graph is also denoted by $G$ and the new vertex is also denoted by $v_{1}$. (The above 3 -step process gives the adjacency matrix $A(G)$ ).

\section{Step-2}

Repeat step-1 until $v_{1}$ is not effectively adjacent to any of the other vertices.

\section{Step-3}

Repeat steps-1\&2 on all the remaining vertices $v_{i}$ of the resulting fuzzy graphs. The final fuzzy graph is (effectively) empty and the number of its (effectively isolated) vertices is the number of effectively connected components of the given fuzzy graph $G$. 


\section{Illustration}

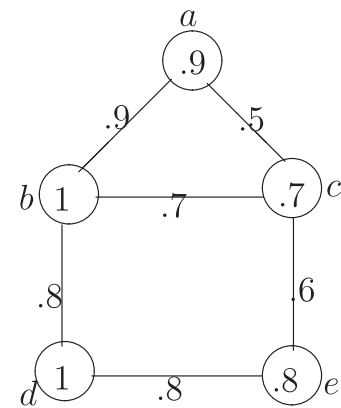

G

\section{Step-1}

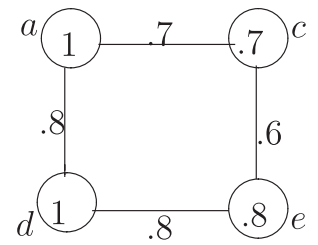

G

\section{Step-2}

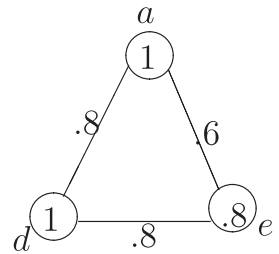

G

\section{Step-3}

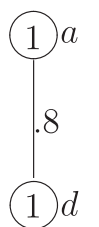

G 


\title{
References
}

[ 1 J John Clark and Derek Allan Holton, A First Look at graph Theory, World Scientific Publishing Co., 1995.

[2] J. N. Mordeson and P. S. Nair, Fuzzy Graphs and Fuzzy Hypergraphs, Physica-Verlag, Heidelberg, 2000.

[3] M. Murugan, Topics in Graph Theory and Algorithms, Muthali Publishing House, Chennai, 2003.

[ 4 ] P. V. Ramakrishnan and T. Lakshmi, Fuzzy Bipartite* Fuzzy Graphs and Powers, International Journal of Mathematics, Game Theory and Algebra, Volume 17 Issue 1/2, pp 7-14, 2007.

[ 5 ] A. Rosenfeld, Fuzzy Graphs, in L. A. Zadeh, K. S. Fu, K. Thanaka and M. Shimura, eds., Fuzzy sets and their Applications to Cognitive and Decision Processes, Academic Press, New York, 1975, 77-95.

[6] A. Somasundaram and S. Somasundaram, Domination in Fuzzy graphs-I, Pattern Recognition Lett. 19 (1998), 787-791.

\author{
School of Mathematics \\ Madurai Kamaraj University \\ Madurai-625 021, India \\ tlakshmi77@yahoo.co.in
}

